ARTICLE

DOI: $10.1057 / s 41599-017-0036-x$

OPEN

\title{
Regenerative medicine: from the laboratory looking out
}

\author{
Jennifer Edwards ${ }^{1}$, Richard Thomas ${ }^{1} \&$ Robert Guilliatt $^{1}$
}

\begin{abstract}
Regenerative medicine has a rich and pluralistic history, characterised by a patchwork of innovation and blind alleys. As scientific researchers, our understanding of this hybrid field of regeneration in a historical and cultural context is far from complete, in part due to the range of non-medical contributory disciplines and a fascination with the future directions of research. This paper explores the different definitions of regenerative medicine and highlights issues faced in regenerative medicine research. We argue that a closer relationship between regenerative medicine and the humanities would enable researchers to better understand the historical context, ethical implications and public perception of this rapidly developing field. In many cases, this would be through better awareness of the existing expertise available in humanities research, which is often not visible to those in the laboratory.
\end{abstract}

\footnotetext{
${ }^{1}$ University of Leeds, Leeds, UK. Correspondence and requests for materials should be addressed to J.E. (email: j.h.edwards@leeds.ac.uk)
} 


\section{Introduction}

wo of the three authors of this paper are scientific researchers working in the field of Regenerative Medicine. They have both worked within the Institute of Medical and Biological Engineering (iMBE) at the University of Leeds, a group whose research focus is to provide ' 50 active years after 50 '. Developments in natural tissue scaffolds, whole joint replacements and better pre-clinical testing strategies are developed to work towards this goal. For the authors, this has included working on development of grafts for skin and ligament repair, development of self-assembling peptides, cell culture, biomaterial scaffolds and mechanical stimulation of cells. This requires a wide range of chemical and biological techniques such as chemical synthesis, microscopy, histology and colourimetric assays to quantify differences in samples. Yet neither of these authors has an undergraduate background in biomedical research; one began in nanotechnology and the other in computer science.

Other members of iMBE come from similar fields of expertise in the physical sciences and engineering, working alongside biologists, biomedical scientists and clinicians. These diverse backgrounds enable the group to bring to bear different techniques, viewpoints and understandings to complex biological and biomechanical problems. This may be to model disease states and treatments, design synthetic or biological replacements, create new drugs or develop cell based therapies. The interdisciplinary environment is essential for success in many of these problems. One problem with this arrangement is that there may be knowledge gaps in the historical or ethical perspective of practitioners in this field. Ethical considerations on the use of human or animal tissue, animal models and the development of medical devices may be unfamiliar to those without a biological background. The authors feel that this range of expertise is undoubtedly necessary for success in developing new regenerative medicine therapies, but we need to do more to understand the wider context of our research. Our field needs to draw on expertise outside of the scientific laboratory.

Regenerative medicine is a multidisciplinary area of research and has evolved as an umbrella subject, covering several wellestablished fields, with a diverse range of meanings and implications. As scientific researchers working in the field, we may define regenerative medicine as the application of scientific principles to repair, restore, supplement or replace the natural function of a biological system. We have derived this definition from that used by the Medical Research Council (MRC) "Regenerative medicine is an interdisciplinary approach that seeks to repair or replace damaged or diseased human cells or tissues to restore normal function" (MRC, 2017). These definitions are forward facing, trying to address an identified issue and meet a clinical need within the scientific landscape. During the recent Past, Present and Futures of Medical Regeneration workshop series (University of Leeds, 2016), discussions covered the meaning of regeneration in a wider context. From a biomedical point of view, the word might suggest the replacement or repair of a lost or damaged part, or natural processes such as natural wound healing or asexual reproduction. Discussions suggested that regeneration, in a generalised sense, may be considered in terms of renewal, rebirth or restoration. The more general view expressed in the workshop made us conscious of what is often missing from the view of modern scientific researchers compared with those in the medical humanities.

Until the early 19th or 20th century, scientists were called natural philosophers and the investigation of the natural world was regarded as part of the same intellectual sphere as the contemplation of moral questions or the exploration of consciousness. The great scientific pioneers of this time associated closely with others working in different fields, including what we now think of as the humanities. Joseph Priestley, credited with the discovery of oxygen, was also a respected theologian, educationist, and political commentator, and was minister of Mill Hill Chapel in Leeds. More recently, scientists like Erwin Schrödinger, a renowned physicist, proposed theories which have helped us make great leaps in biology. Schrödinger's lecture series entitled 'What is Life?' was later reproduced as a book and has been credited with aiding James D. Watson and Francis Crick in their discovery of the structure of DNA (Derry, 2004). This sort of close contact between disparate fields and disciplines is often missing from modern scientific research groups.

The phrase 'regenerative medicine' first appears in the scientific consciousness in 1992, where it was used to describe a "new branch of medicine that will develop, that attempts to change the course of chronic disease and in many instances will regenerate tired and failing organ systems" (Kaiser, 1992). Since then, there has been an exponential increase in the number of journal articles and research projects identifying themselves as regenerative medicine. Such projects often seek to repair or replace damaged or failing organs or tissues to meet a clinical need, extend life or improve quality of life. Given the long history of medical research, there was already a vast body of work into improving or extending human life, which can now be considered as falling into the area of regenerative medicine.

The first recorded examples of reconstructive surgery using skin grafts were written by Sushruta, dubbed the 'father of surgery' in India, in his text the Suśruta-samhitā around 750-800 B. C. In 1869 Jacques-Louis Reverdin would, incorrectly, claim to be the first to report a successful skin graft (Davis, 1941). The evolution of cell biology and a detailed understanding of the structure and function of skin have led to the development of cell based therapies for treating skin trauma. Keratinocytes, cells which form the upper layers of the skin and are responsible for its important barrier function, were first reliably cultured in the laboratory in 1975 by Rheinwald and Green (Rheinwald and Green, 1975). This lead to the production of sheets of keratinocytes for clinical use in burns victims in the 1980s (O'Connor, 1981, Gallico et al., 1984). Today this approach would be considered a tissue engineering and regenerative medicine strategy, and many attempts are being made to further develop and improve this treatment approach.

Closely linked to regenerative medicine research is the early work on embryonic development and wound healing in animals. The work of pioneers like Wilhelm Roux and Hans Driesch in embryo development investigated how cells responded to damage during development, which could be considered as the historical origin of both regeneration and stem cell research. In 1981 the first pluripotent stem cells were isolated and cultured from mouse embryos, but as far back as the 18th century, descriptions of the regenerative properties of animals were formulated: Reaumur detailed the regenerative properties of crustaceans, Spallanzani studied the regeneration of tadpole tails and Singer observed the regeneration of limbs in newts (Dinsmore, 2008). The work into the regrowth of limbs in amphibians is more easily recognised as the inspiration behind regenerative medicine research and still forms a large part of current scientific study.

\section{Challenges in regenerative medicine research}

For many early career scientific researchers in regenerative medicine, there may have been no formal training in medical history, ethics or philosophy. Such topics may not be considered important initially as they are not used directly to inform the conduct of research immediately post- $\mathrm{PhD}$. This deficiency may be problematic when considering the translation of research from 
bench to bedside. Whilst working in the laboratory, it is easy to lose sight of how the research will be used in the real world and how it may be perceived by a non-specialist audience. We argue that a greater understanding of the research carried out by humanities scholars would go a long way towards addressing these gaps in knowledge. To this end, we will here seek to introduce a range of problems encountered by regenerative medicine researchers, where the skills of humanities scholars have the potential to improve scientific practice.

History. Historically, failure in science was as important as success, however in modern research this idea has arguably been lost. While there is some scope for negative data to be published (such as The Journal of Negative Results in Biomedicine or Journal of Articles in Support of the Null Hypothesis), negative or inconclusive data generally has a low priority for publication. This means that there are swathes of relevant research which remains unpublished due to perceived failure. Although there are efforts to address this (such as creating data repositories for research council funded research), it is far from common practice. This situation has come about due to the dual effect of modern journals and the increase in incremental directional research funding. Data which is positive, and so changes our perception of a topic or conclusively demonstrates a new behaviour, can be published with relative ease. Negative data, even that which has the same potential to change perceptions, is often inaccessible to most researchers. Most concerns regarding the quality and significance of research data focus on rooting out misconduct rather than questioning the epistemological assumptions of data production (Hiney, 2015, Martinson et al., 2005). This is not to say that philosophers and sociologists of science have not identified these issues (Knorr-Cetina, 1983, Knorr-Cetina, 1991) but rather that their insights rarely seem to reach the lab.

The advent of the internet has also created a divide between recent and historical research. Research published before the rise of modern electronic journals is significantly more difficult to access than recent publications. This landscape of missing or incomplete information can lead to the repetition of research which has already been carried out. It leaves gaps in our understanding of a process or disease, by reducing the visibility of proved and disproved hypotheses. In some cases, it makes it difficult for researchers to access the theory behind a technique and understand how it may interact with a given sample. A large amount of research conducted within laboratories is based upon well-established techniques and protocols. The use of standardised protocols is common in research but also forms a critical part of the Good Manufacturing Process (GMP), which is essential for gaining regulatory approval. Standardised protocols carry their own risks, as they are easy to follow without understanding their underlying principles. Regenerative medicine is predominantly focused upon addressing clinical need and scientific researchers are always focused upon the next idea, solution or breakthrough and what this will mean to the future. This is a symptom of the short-sighted nature of research, sometimes due to the short-term nature of postdoctoral research positions. This situation and the attitude that historical research is less informative shows a kind of arrogance in the modern researcher, the assumption that the best and most relevant papers to read are the most recently published. Even among researchers with an interest in the history of their field, this short-sightedness can in turn lead to a teleological understanding of the field's development-a 'presentism' that imagines said history as a series of advances towards the standard practice of today-and a failure to think critically about whether current practice and best practice are the same thing.
Interacting with medical historians would go a long way towards addressing this. The perspective offered by medical historians would allow researchers to better ask if the research has already been conducted, if there are gaps that modern techniques can fill in pre-established theory, if explanations to issues faced in the laboratory have already been discussed, or if a consideration of historical practices can prompt a reappraisal of current ones. In laboratory research, there is rarely any doubt that positive results in the lab will have positive results for patients and society. As researchers, we would like to believe that this is nearly always true, but we rarely consider that it might not be. Not only does this mean that cultural and social issues are not considered, but it may blinker the research from new avenues or useful methods from other research areas in a drive to answer a very specific research question.

Research developed within the laboratory should, where possible, be translated into a form which can be used in the real world to improve the lives of patients. This is an expensive, complex process which is often unsuccessful. Following basic development in the laboratory, it is necessary to carry out proof of concept work, animal studies and develop GMP compliance to demonstrate the safety and efficacy of the research before the start of basic clinical trials in humans. New treatments are developed to treat patients, and it is important that we minimise the side effects and complications from the treatment. Experimentation on humans to understand the basic science is considered unethical, drawing to mind experiments carried out on unwilling subjects in the past. Medical trials differ from experimentation, as there is a need to prove the treatment is likely to be safe for human patients, prior to demonstration of improvement over existing treatment options. The concepts behind medical trials are ancient, and the first medical trial as we would recognise it today was conducted in 1747 (published 1753) by James Lind (Hughes, 1975), who tested treatments for scurvy. In 1801 John Haygarth demonstrated the importance of a control group in medical trials to account for the placebo effect (Haygarth, 1800). Many of the concepts and ideas developed historically in medical trials are now standard practice. Unlike the majority of basic research, clinical trials are subject not only to scientific input but also ethical and cultural considerations. There is a need for them to address not only the effectiveness and safety of a new treatment, but the value to society, and whether or not it will be effective outside of the trial methodology. Many researchers have concerns about extrapolating from trial data to treat individual patients, each of whom have a unique set of conditions (Kent and Hayward, 2007). In some trials, such as those in emergency medical situations, it may also be difficult to ensure that the desired protocol is adhered to, reducing the clarity of the findings (Sanders, 2011). Clinical trials must consider input from stakeholders who have different priorities, such as patients, practitioners, regulatory bodies and industry. Will and Moreira (2016) discuss the changing role of Randomised Controlled Trials (RCTs) as an integral part of drug research and development. The volume discusses the challenges in running RCTs from finding suitable methodologies, navigating regulatory constraints and changing societal views. As part of this, they explore inherent bias in reporting; if a drug does not have the desired effect, this may not be reported, leading to missing information and future waste in resources on similar drugs. In other cases, trials may show benefits of the control treatment, but these may not be translated into general practice if they are not clearly reported. Recruitment of patients to trials may also be affected by the healthcare systems in place; countries without public health services may recruit participants who see a trial as their only means of treatment. Their work highlights the need for trials to consider factors other than the direct outcomes of a treatment, such as patient and 
practitioner experiences and attitudes and how clinical research links to public health policy. An understanding of the mistakes of the past may make it easier to develop scientific research which addresses similar issues from the start. Rather than training scientists in a limited way to understand the ethical and cultural aspects of medical research, it would be more effective to bring in existing expertise from humanities scholars.

Critical reflection. The ability to critically analyse the literature is a skill developed at undergraduate level which remains important throughout a scientific career. Research students are taught to assimilate published research to inform their own experiments and understand the context of their project. These critical analysis skills are often not applied in other situations, such as to the methodologies used during research. In partnership with the Leeds University Business School, the iMBE allowed groups of its researchers to enrol on a postgraduate course in Professional Innovation Management. As well as discussing the common view of innovation as the creation of new technologies, the course aimed to provide researchers with the skills to be innovative in their research methodologies. It involved practicing a range of critical analysis and critical reflection techniques to consider inefficiencies in their current work. The tools provided felt very unfamiliar at the start of the course, and many students did not see how reflecting on their feelings in a given situation within the lab would help inform their work. Developing these skills in researchers more widely would allow them to better consider other perspectives to challenge their own perceptions, but they would still be constrained by their area of expertise. Such skills are being introduced to the next generation of scientists at undergraduate level, such as through assessment of reflective journals during final year project work, but there are many established researchers who will not have this experience. Encouraging the involvement of humanities researchers trained in these skills to advise on laboratory research methods may help provide a wider perspective.

One of the areas where a different perspective would be of great benefit to researchers in regenerative medicine would be to improve understanding of patient expectations. As researchers, we often aim to restore normal function in the damaged tissue; in many cases patients primarily want relief from pain, as this can be the most limiting factor in their daily lives. An example of this is total hip replacements (THRs), where the device is designed to restore normal function and different combinations of materials may even allow the patient to take part in high-impact activities. Since their development following World War II, hip prostheses have undergone many developments in material, manufacture and surgical procedure. There has also been a huge change in the expectations of patients and surgeons around the outcomes of these operations, discussed in detail by Anderson et al. (2007). Initial expectations of pain relief have been replaced by a desire to return to normal activities, as well as a shift towards younger patients requiring longer lasting prosthesis and perhaps several revision surgeries. The book also explores the changing nature of information provided to patients, from leaflets written by surgeons (covering the disease, procedure and hospital stay) to those produced later with patient input or even entirely by patients. This shift in style provides more information to the patients on the risks, as well as the impact on many aspects of their daily lives, information they may not discuss openly during surgical consultations. This dialogue is still far removed from the world of the laboratory researcher who may be developing the materials or prostheses. The exposure to a range of views would benefit the researcher by providing different perspectives and allow patient benefit to be better addressed along the development pathway. Depending on the research environment, there may be direct contact with clinicians and patients during research design and development. Within NHS research institutions, there is a strong focus on patient and public involvement (PPI) with research, from deciding which research themes to prioritise through to study design, evaluation and reporting. Outside of research in NHS institutions, this interaction may be limited to the people who obtained funding, and is not always available to postdoctoral researchers. Raising the awareness of medical history texts exploring PPI may provide laboratory researchers with an insight into the importance of considering how technologies may affect the patient, far removed from the laboratory.

Ethics and culture. Considering the ethical implications of a given scientific technique, treatment or clinical trial is another area which would often benefit from the perspective of someone working outside the field. Animal and human tissues are often used in a laboratory setting for a wide range of applications, such as the isolation of cells for use in basic research. For many researchers this will represent the first encounter with the real world application of ethics and working to established regulations. Without detailed knowledge of the ethical arguments for a particular regulation, or involvement in the preparation of ethics agreements for research, scientists may fail to grasp what the regulations are designed to protect. One area of research in regenerative medicine revolves around processing natural tissues (such as skin or tendon) to remove the cells. This leaves a matrix of proteins and biologically active molecules, which can be recognised by the body. While the cells within a tissue are unique to an individual, the rest of the tissue is highly similar between different people and even different species. If the cells are removed, the question can be raised as to whether the tissue still belongs to a particular donor or animal-when is a pig not a pig? While researchers may look upon the use of animal tissue as necessary to maintain a supply of suitable graft material, there are many situations in which this may not be appropriate. Patients may have religious objections to donated tissue, whether the source is animal or human, or it may only be problematic from a particular animal. These viewpoints are important to consider when designing the initial programme of research, to ensure that it has the potential to form a useful therapy. Researchers may not be equipped to answer questions like these, such as does the perception of a treatment change when it is a lifesaving technology compared to one which only improves the quality of life? These sorts of questions are also important in the regulation of new technologies for human benefit which use animal products. From the early days using irradiated mouse feeder cells to produce skin grafts from human cells, to the use of animal derived matrices, regulations have evolved to prevent cross-species disease transmission (Michael et al., 2006). Such regulations could provide a glimpse into the practical implications of these questions, but relatively few within the laboratory consider the route to market of their work during development.

At the fringes of current research, the biggest dreamers are starting to investigate what most would consider science-fiction. Advances and developments in areas such as cybernetics, cryopreservation, regeneration and rejuvenation have the potential to change all aspects of society. It is our belief that the implications of this research should be looked at pre-emptively rather than in a reactionary manner. Indeed, such research is being carried out already by numerous researchers in philosophy, for example the ethical issues raised in Savulescu and Bostrom's edited collection, Human Enhancement (2009) or Jérôme Goffette's concept of 'anthropotechnics' (a distinction between medical 'repair' and augmentation) (Goffette, 2015). To allow the social, economic, and cultural implications of scientific advances 
to be discussed before they become a reality, research scientists must actively engage with the wider academic community to develop and advise on legal and ethical frameworks to cover future developments. It may also provide insights into how new technologies may be used or misused, the fears that could be played upon in the public mind, and suggest new uses that the developers have never considered. We would argue that the only way for such a culture to develop is for regular dialogue between disciplines, and that a regular forum for these discussions must be established to overcome the problems encountered when every new initiative in this area is forced to 'reinvent the wheel' of science-humanities communication.

The cultural and ethical questions raised by new research ideas require consideration and debate before a new treatment enters the clinical setting. An example of this can be seen in the history for the treatment of diabetes. For many years, insulin-dependent diabetic patients would have received animal source insulin from pigs, cows, horses or fish. Whilst this treatment worked well and was responsible for saving many lives, impurities in the extracts meant different batches of medicine did not show the same effectiveness. The first instance of genetic engineering was used to create a synthetic version of human insulin in 1978, which came to market in 1982 (Quianzon and Cheikh, 2012). Synthetic insulin can be produced to a much higher standard of reproducibility and is now the source for $70 \%$ of patients around the world. Using a synthetic analogue of human insulin also means that the treatment is suitable for those with ethical objections to the use of animal products as medicine. This technique uses genetic modification to produce a lifesaving drug based on the human insulin molecule. This case demonstrates not only the ethical considerations that can face medical research but also how such considerations can be used to drive research and development, leading to innovation with a further reaching impact.

The use of animal models in research is another area of intense ethical debate. Animals have been used for millennia to try and develop our understanding of biological processes. Early work on anatomy was often conducted using a range of animals, which led to many misunderstandings and incorrect assumptions about human anatomy. One of the most famous examples of this is in the works of Claudius Galenus (A.D. 131-192). Galen misrepresented the shape of the human heart, the location of the kidneys, the shape of the liver and he stated that rete mirabile, at the base of hoofed animal's brains, was present in humans (Malomo et al., 2006). Galen made these mistakes because he relied on animal dissection, as human dissection was not only taboo but illegal. Galen was revered and, since the taboo persisted, many of his erroneous ideas were perpetuated and the field of anatomy advanced slowly until the sixteenth century. There are now many animal models of human disease, which are used to develop new treatments. The use of such models can provide insight into disease progression or understanding of how a new drug interacts with the body. Since the latter part of the 20th century, there has been a drive to reduce the reliance on these models in research, due in part to recognising the disparity between results in animals and outcomes in human patients (van der Worp et al., 2010). Such discrepancies may arise due to interspecies variability in molecular processes, or differences in the way a particular disease affects humans. In addition to scientific limitations, there are many ethical objections to the use of animals in research. Concerns over the animal welfare have led to many strict guidelines on the care and handling of animals and more rigorous study design to ensure that the minimum number of animals are used to provide a conclusive research outcome.

In the UK, the NC3Rs (National Centre for Replacement, Refinement and Reduction of Animal Models in Research) was set up to promote reduction and refinement in the use of animal models (NC3Rs, 2017). The ethical objections and inadequacies of animal models are a driver of new research that is changing the face of regenerative medicine and medical diagnostics. People who choose to work in the field of regenerative medicine have widely accepted that at some point animal products, tissues or models will be necessary to supplement our understanding. This wide acceptance leaves very few people who are actively engaged with this field who can voice the opposite opinion; interaction with people outside of the research setting not only provides researchers with the impetus to critically evaluate what we are doing and what we need to learn from any animal work. Working to reduce the reliance on animal models for research has already led to the emergence of new research areas. For example, the development pathway for the growth of whole organs for transplant has led to the development of small model tissues that can be used in the creation of so-called "organ on a chip" technologies (Bhise et al., 2014). Such technologies may eventually offer a comprehensive alternative to animal models of drug interaction.

Communication. One of the most daunting areas facing a scientist is communicating the importance of their research. While we may believe strongly in what we are doing, it can often be difficult to express this in a format which is understood by a nonspecialist. Grant awards often now require demonstration of research impact and public engagement. Communication of complex ideas is not only an important skill when applying for funding, it is becoming more important than ever to communicate research to a wide, public audience. More than demonstrating impact and dissemination of findings to public stakeholders, it is important to inspire not only the next generation of scientists, but people of every age to engage more with the advances in medical treatments that may 1 day be available to them. The Royal Society has been organising public lectures for general audiences for over 200 years. The Christmas lecture series was founded in 1825, to bring complex scientific concepts to young people, a tradition which continues successfully to this day. Many researchers still do not take part in events which engage the public about their research for a variety of reasons, often including a perceived lack of relevant skills. Interaction with the public can be daunting as it requires the scientist to leave the comfort zone of their technical language and express complicated concepts in a simple manner. Specific terminology may be indecipherable to researchers outside of a specific field, let alone to a non-scientist. Persuading researchers to avoid acronyms or that having to use a short phrase instead of a single scientific term can be difficult. Other terms may have different meanings in the laboratory to in general use. One good example is the word 'theory'-in a scientific context, a theory is an explanation whose predictions have been verified by experiments or other evidence, whereas in colloquial speech a theory is a conjecture or guess. The most famous example of this is the theory of evolution, which is supported by a huge body of scientific research and understanding, but is often dismissed, by its detractors, as 'just a theory'.

Breaking these language barriers can have benefits for the researcher, which many do not recognise until confronted with the situation. Being able to explain your research in simple terms requires you to have a greater understanding of your work and its context. The benefit of working with literary colleagues in this is to ensure that the language does not become too simplistic as to become a different type of incomprehensible. To demonstrate this, Theo Sanderson challenged people to describe their job using only the 1000 most common words using an online text editor (Sanderson 2013). This was inspired by an issue of the xkcd 


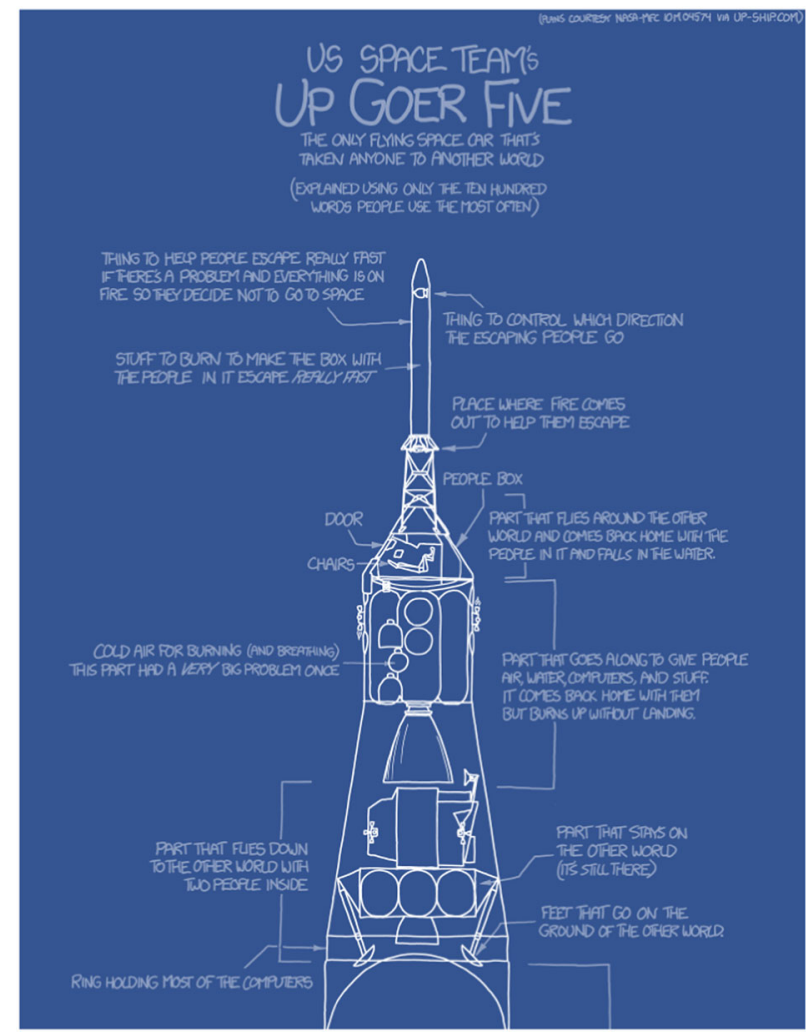

Fig. 1 The dangers of oversimplification of language when describing scientific equipment. Partial reproduction of issue 1133 of the xkcd webcomic (https://xkcd.com/1133/), which led to the creation of the UpGoer Five text editor (http://splasho.com/upgoer5/). Image reproduced under the Creative Commons Attribution-NonCommercial 2.5 License, as detailed on the author's website (https://xkcd.com/license.html)

webcomic (Munroe 2013), explaining the Saturn V rocket using only these 1000 words (Fig. 1), demonstrating clearly that using only common words does not necessarily lead to clear explanations. Since the creation of an online text editor for the challenge, hundreds of scientists have attempted to explain their research with these limited terms, and some of the responses are available on the website. While the challenge of translating your work into these simplistic terms is a useful exercise in identifying overly technical language, it is very restrictive and leads to equally unhelpful explanations. Working closely with authors could help scientists think about their language, without 'dumbing down'. Working closely with visual artists has the potential to convey difficult concepts without words at all. A technique developed to demonstrate bacterial evolution for undergraduate teaching at Harvard was later used in research to demonstrate the rapid evolution of antibiotic resistance (Baym et al., 2016). The associated video quickly caught the attention of the media and presented a minor victory in science communication (Pesheva, 2016). If science researchers were supported in building longlasting partnerships with artists, such 'viral' successes might become commonplace. Scientific communication should be used to inspire and inform, and it is critical to ensure that the language or expression used is not condescending. There are also many opportunities to help create other artistic works inspired by science, either through public or professional collaborations. This enables researchers to display their scientific work, even if they do not feel that they have the required skills. There is a growing interest in these sorts of collaborations, with funding bodies such as the Wellcome Trust creating funding opportunities. Encouraging scientific researchers to engage with these schemes, which may take time away from their experimental research, is key to ensuring their success.

Funding. Scientific research is carried out within the constraints of strict budgets and new research ideas are explored subject to the acquisition of suitable funding. For many research councils, there are specific research priorities which they are interested in funding. Researchers must therefore clearly focus their research to fit within these themes, which can greatly restrict the areas of exploration available to them. While there are large programme grants which allow for more open research directions, these are only available to those with well-established careers and publication records. The nature of this research landscape means there is now minimal funding available for 'blue sky' research, which may prevent serendipitous discovery as has been seen many times in the past. Whilst regenerative medicine researchers believe that their work will have huge benefits for society and patients a lack of understanding of healthcare economics and community services means that this may not always be the case. In many cases, translation of a new technology from a research environment to the clinic fails, which may be influenced by a variety of different factors. The problems of implementation and adoption are numerous, but beyond the scope of this paper. The authors recognise, however, that these issues are taken seriously by health researchers (examples include Portela et al. (2015) and Jones et al. (2014)).

An understanding of community support is also important; if new regenerative therapies require a high level of assistance, physiotherapy or monitoring by experts, the required support structures may not be in place for the technology to be a success. Input from those who understand how health economics, community support and patient aftercare work before research reached clinical trials could raise potential issues earlier in the development pathway. While there are many researchers working in these fields, they are often not involved in research until it leaves the laboratory and is working towards clinical trials. This means that significant time, effort and resources may have been invested in something which will not work in a practical setting. Contact with patients is also important to understand whether a particular technology or therapy would be practical in their daily lives.

\section{The way forward}

To try and address some of the problems introduced throughout this paper, it is increasingly apparent that we need better communication and knowledge transfer across the whole spectrum of research in regenerative medicine. We believe that closer relationships between regenerative medicine and the humanities would enable researchers to better understand the historical context, ethical implications and public perception of this rapidly developing field. This has the potential to influence research decisions, improve communication between researchers, healthcare practitioners and the public and encourage a wider engagement with the cutting edge of biomedical research. Laboratory researchers in regenerative medicine face many challenges not directly relating to their research. These can include developing an understanding of the ethical or cultural implications of their work, communication challenges or critical reflection. Lack of exposure to other viewpoints may make it difficult for scientists to understand how their research may be viewed in a negative light, as they are driving to make a positive difference. In other cases, a lack of access to the clinical setting may prevent researchers from developing technologies which can be successfully translated into new treatments for patients. Engaging with researchers outside of the scientific world who 
have skills and understanding in these areas could be beneficial for research development and perception outside of the lab. Whilst preparing this article and considering areas in which we (as early career scientific researchers) felt underprepared, it became clear to us that there were many existing experts in these areas. For example, the Centre for History and Philosophy of Science at the University of Leeds was within easy reach but unknown to us until a late stage in writing. The biggest hurdle is ensuring better transfer of knowledge and expertise between the groups.

There are many possibilities which could help encourage and normalised such collaborations. The scientific authors of this paper found the Past, Present and Futures workshop to be a very positive experience, which led to their desire to write this article. Such discussions of what our goals as laboratory based regenerative medicine researchers and what the terms mean to scholars outside this world provide an opportunity for reflection on our aims and understanding. To encourage such interaction from a wider range of scientists, it may be interesting to look at lectures or seminars, presented concurrently by both scientists and humanities scholars. Many departmental seminar series are well attended by those within the school, and these may be a good place to introduce wider concepts to the audience.

Engagement with the humanities has the potential to drive academic development. New concepts are often explored in literature and popular culture before or at the point of new scientific developments. A prime example of this is the impact that Star Trek has had on our lives. Dr Martin Cooper, inventor of the first handheld mobile phone, credits the original series communicator as his inspiration. Star Trek has also had a huge impact on medical research goals such as the X-price to develop a handheld medical diagnostic device akin to the tricorder. Current medical developments such as 3D printing are finding their way into popular culture; FRANKeN5Te1N, a 2015 modern retelling of Mary Shelley's novel, has the monster 3D printed rather than assembled from the graveyard.

To foster a more visible literary understanding of the regenerative medicine research, the ideas of writers in residence within large research groups has considerable merit. Immersing a writer in the world of research will allow them to bring an outside perspective to the researchers, as well as spending time understanding how the laboratory functions. This would allow opportunities to question scientific practices and see how time and resources can affect the scientific process. Discussion of these processes with someone from outside their professional sphere could help scientists critically reflect on their work and help them understand outside perspectives. For these sort of collaborations to function, there would need to be input from the scientific funding streams to include salary for the humanities researcher or creative artist. This could be to cover a short-term secondment or a longer term residence. A longer term style of collaboration may also help scientists acclimatise to working closely with humanities researchers from $\mathrm{PhD}$ students upwards, placing this as a normal activity in their future careers. Depending on the specific collaboration, it would also allow scientists time to immerse themselves in the world outside the laboratory and gain further insight into ethics, cultural or healthcare research. Collaborations such as these could be designed to ensure impact for the research, through better communication, production of cross-disciplinary works or personal development of researchers. Such schemes could encourage the production of new materials and exhibits to start discussions around scientific advance and research.

While public engagement with research is now being driven forward, partly due to the expectations of research councils, it has a much wider potential. Involvement of ethicists and cultural studies researchers could help move scientists away from the simple attitude of 'tell the public what you did with the money they gave you'. This could be to engage the public in debates, allowing their opinions and concerns to reach the scientists while they conduct the research. Such debates can be of great value to the researcher, forcing them to think about their research in less technical language and solidifying their knowledge to respond to questions from outside their limited scientific circle. One driver of engagement with schoolchildren is to instil in them an interest in science and to encourage the idea that it is not just for the geeks. It is also useful to encourage them to think critically about the media's portrayal of scientific development and our own role in allowing this portrayal-have we been doing enough to ensure that our science does not seem aloof and suspicious? This interaction at an early career stage may help equip the next generation of principal investigators with the skills to consider the wider implications of their research and give them the confidence to engage with non-scientists. Even if young researchers are not currently developing their own research with a view to translation, knowledge of the skills and expertise available to them outside of their field could make an enormous difference when they gain independence. Many junior researchers do not realise the range of studies outside of science which can inform development and translation.

Perhaps the most important reason to encourage these collaborations is to help make them a normality. Regenerative medicine research should not be secluded, as it is carried out with the end goal of providing benefit to patients. Encouraging collaboration between those who research the cultural, social and ethical effects of research and those who perform the research would help drive research forward in a manner which is useful to society.

Received: 16 June 2017 Accepted: 26 October 2017

Published online: 14 November 2017

\section{References}

Anderson J, Neary F, Pickstone J (2007) Surgeons, manufacturers and patients: a transatlantic history of total hip replacement, Springer, London

Baym M, Lieberman TD, Kelsic ED, Chait R, Gross R, Yelin I, Kishony R (2016) Spatiotemporal microbial evolution on antibiotic landscapes. Science 353:1147-1151

Bhise NS, Ribas J, Manoharan V, Zhang YS, Polini A, Massa S, Dokmeci MR, Khademhosseini A (2014) Organ-on-a-chip platforms for studying drug delivery systems. J Control Release 190:82-93

Davis JS (1941) Address of the president: the story of plastic surgery. Ann Surg 113:641-656

Derry JF (2004) Book review: what is life? Human Nat Rev 4:124-125

Dinsmore CE (2008) A history of regeneration research: milestones in the evolution of a science. Cambridge University Press, Cambridge (England); New York

Gallico 3rd GG, O’Connor NE, Compton CC, Kehinde O, Green H (1984) Permanent coverage of large burn wounds with autologous cultured human epithelium. N Engl J Med 311:448-451

Goffette J (2015) Enhancement: why we should distinguish anthropotechnics from medicine. Inquiring into human enhancement. Springer, London

Haygarth J (1800) Of the imagination, as a cause and as a cure of disorders of the body; exemplified by fictitious tractors, and epidemical convulsions. In: Edinburgh, RCOPO (ed) Read to the literary and philosophical society of Bath. Bath: printed by R. Cruttwell, London

Hiney M (2015) Research integrity: what it means, why it is important and how we might protect it. In: Strasbourg (ed) Science Europe

Hughes RE (1975) James Lind and the cure of scurvy: an experimental approach. Med Hist 19:342-351

Jones E, Lees N, Martin G, Dixon-Woods M (2014) Describing methods and interventions: a protocol for the systematic analysis of the perioperative quality improvement literature. Syst Rev 3:98

Kaiser LR (1992) The future of multihospital systems. Top Health Care Financ $18: 32-45$ 
Kent DM, Hayward RA (2007) Limitations of applying summary results of clinical trials to individual patients: the need for risk stratification. JAMA 298:1209-1212

Knorr-Cetina KD (1983) The ethnographic study of scientific work: towards a constructivist interpretation of science

Knorr-Cetina KD (1991) Epistemic cultures: forms of reason in science. Hist Polit Econ 23:105-122

Malomo A, Idowu O, Osuagwu F (2006) Lessons from history: human anatomy, from the origin to the renaissance. Int J Morphol 24:99-104

Martinson BC, Anderson MS, de Vries R (2005) Scientists behaving badly. Nature 435:737-738

Michael M, Brown N, Faulkner A, Kent J (2006) Regulating hybridity cleaning up and making a mess in the leaky worlds of xenotransplantation and tissue engineering. Social Theory \& Health 4(1):1-24

MRC (2017) Regenerative medicine and stem cells. Medical Research Council. https://www.mrc.ac.uk/research/initiatives/regenerative-medicine-stem-cells/. Accessed 2 Jun 2017

Munroe R (2013) Up goer five. https://xkcd.com/1133/. Accessed 3 Jun 2017

NC3RS (2017) National centre for the replacement, refinement and reduction of animals in research. https://www.nc3rs.org.uk/the-3rs

O’Connor NE, Mulliken JB, Banks-Schlegel S, Kehinde O, Green H (1981) Grafting of burns with cultured epithelium prepared from autologous epidermal cells. Lancet 1:75-78

Pesheva E (2016) Bugs on screen. https://hms.harvard.edu/news/bugs-screen. Accessed 4 Jun 2017

Portela MC, Pronovost PJ, Woodcock T, Carter P, Dixon-Woods M (2015). How to study improvement interventions: a brief overview of possible study types. BMJ Qual Saf 25(5):325-336

Quianzon CC, Cheikh I (2012) History of insulin. 2012:2

Rheinwald JG, Green H (1975) Serial cultivation of strains of human epidermal keratinocytes: the formation of keratinizing colonies from single cells. Cell $6: 331-343$

Sanders AB (2011) Cardiac arrest and the limitations of clinical trials. Mass Medical Soc. New England Journal of Medicine 365(9):850-851

Sanderson T (2013) The up-goer five text editor. http://splasho.com/upgoer5/. Accessed 3 Jun 2017

Savulescu J, Bostrom N (2009) Human enhancement. Oxford University Press on Demand, Oxford van der Worp HB, Howells DW, Sena ES, Porritt MJ, Rewell S, O’Collins V, Macleod MR (2010) Can animal models of disease reliably inform human studies? PLoS Med 7:e1000245

Will C, Moreira T (2016) Medical proofs, social experiments: clinical trials in shifting contexts. Routledge, Oxford

\section{Data availability}

Data sharing not applicable to this article as no datasets were generated or analysed during the current study.

\section{Additional information}

Competing interests: The authors declare no competing financial interests.

Reprints and permission information is available online at http://www.nature.com/ reprints

Publisher's note: Springer Nature remains neutral with regard to jurisdictional claims in published maps and institutional affiliations.

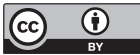

Open Access This article is licensed under a Creative Commons Attribution 4.0 International License, which permits use, sharing, adaptation, distribution and reproduction in any medium or format, as long as you give appropriate credit to the original author(s) and the source, provide a link to the Creative Commons license, and indicate if changes were made. The images or other third party material in this article are included in the article's Creative Commons license, unless indicated otherwise in a credit line to the material. If material is not included in the article's Creative Commons license and your intended use is not permitted by statutory regulation or exceeds the permitted use, you will need to obtain permission directly from the copyright holder. To view a copy of this license, visit http://creativecommons.org/ licenses/by/4.0/.

(C) The Author(s) 2017 